\title{
Commentary More Than Just Chillin': Interactive Effects of Menthol and Nicotine in Drug Reward
}

\author{
Valeria Lallai' and Christie D Fowler, \\ 'Department of Neurobiology and Behavior, University of California Irvine, Irvine, CA, USA
}

Neuropsychopharmacology (2017) 42, 2283-2284; doi:I 0.1038/npp.2017.107; published online 5 July 2017

'...it appears that nicotine and menthol combine in an additive manner to determine degree of impact' -Philip Morris Internal Memo, Gullota et al (1989)

Menthol is a common additive found in varying concentrations in most tobacco cigarettes, as well as a flavorant in electronic vapor cigarettes (e-cigarettes). The impact of flavorants in promoting initial use and later dependence has been a concern since shortly after their introduction into the marketplace. Indeed, the potential for menthol to interact with nicotine and thus promote dependence was noted by the cigarette industry over 25 years ago, with subsequent product development efforts focused on exploiting this interaction to increase user consumption (Gullota et al, 1989). More recently, population-based studies have established that a large proportion of youth preferentially consume flavored tobacco products, with estimates as high as $80 \%$ (Villanti et al, 2017). Moreover, adult women and racial/ethnic minorities, including African Americans, Latinos, and Pacific Islanders, disproportionately consume mentholated nicotine products (FDA, 2013; Villanti et al, 2017). While this increased consumption by some populations has been proposed to be due to focused marketing in targeted communities, genetic/biological factors (eg, differences in drug metabolism or receptor expression) may also underlie an increased vulnerability for dependence.

Traditionally, menthol has been considered non-harmful based on its common use in a variety of consumer products (lip balm, cough medication, topical analgesic, mouthwash, and food flavorant) and relatively non-toxic effects (FDA, 2013). Menthol's characteristic cooling sensation has been attributed to increased $\mathrm{Ca}^{2+}$ influx through activation of the TRPM8 thermoreceptor, a mechanism that has been proposed to counteract the aversive oral properties of nicotine and thus promote palatability and consumption

\footnotetext{
*Correspondence: Dr CD Fowler, Department of Neurobiology and Behavior, University of California Irvine, 1232 McGaugh Hall, Irvine, CA 92697, USA, Tel: +l 949824 8363, Fax: + I 9498242447 , E-mail: cdfowler@uci.edu

Received 28 April 2017; accepted 3 May 2017
}

(Fan et al, 2016). However, emerging evidence has led to a reassessment of the impact of menthol on addiction processes in consideration of the documented central effects on cholinergic signaling mechanisms. For instance, chronic menthol treatment, in the absence of nicotine, has been shown to induce an upregulation in nicotinic acetylcholine receptors (nAChRs) in GABAergic midbrain neurons and to alter the stoichiometry of subunit expression by stabilizing lower sensitivity receptor subtypes (Henderson et al, 2016).

In this issue of Neuropsychopharmacology, Henderson et al (2017) provide evidence to support the hypothesis that menthol and nicotine act synergistically to upregulate $\mathrm{nAChR}$ expression in dopaminergic neurons; effects that are proposed to mediate a functional enhancement in neuronal excitability. These findings represent a significant advance in our understanding of how both nicotine and menthol may act together to mediate nicotine addiction. In these studies, menthol and nicotine co-administration induced a conditioned place preference (CPP) for a subthreshold dose of nicotine and potentiated CPP expression for a rewarding dose of the drug. However, administration of menthol alone did not result in the development of a CPP. Thus, these data suggest that nicotine and menthol interact synergistically to drive reward-related behaviors. It is worthwhile to note that in their prior study, chronic treatment with menthol alone prevented the formation of a CPP for nicotine (Henderson et al, 2016). While these data may initially seem contradictory, the duration of exposure and pharmacokinetics associated with acute co-administration likely mediated the differential reward-related effects. In the prior study, menthol was administered continuously in a minipump for 11 days and during CPP training. Thus, the continuous baseline level of menthol exposure may have altered circuit responsivity during both the nicotine and vehicle training sessions. In contrast, the current study co-administered nicotine and menthol acutely during CPP training (Henderson et al, 2017), thus providing a more accurate assessment of co-exposure similar to that found with nicotine product consumption in humans. Another strength of the current study is reflected by the fact that the authors have taken into account both the ratio of menthol to nicotine in a typical mentholated cigarette and 
respective brain penetration levels. Given these important considerations, the current approach offers a rationale foundation by which the findings support translational relevance to human smokers. Finally, since both menthol and nicotine were administered peripherally, rather than orally, the rewarding properties cannot be attributed to the buccal effects of either substance in the current study, thus providing a distinct advantage when attempting to disentangle sensory $v s$ central effects of menthol and nicotine action.

In consideration of the potential effects of menthol on other receptor mechanisms, such as TRPM8, the authors extended their investigations to demonstrate the direct effects of menthol and nicotine on $\mathrm{nAChRs}$ in dopaminergic neurons. In these studies, midbrain cultures from tyrosine hydroxylase-eGFP mice were examined to assess electrophysiological changes in dopaminergic neuronal activity. Chronic treatment with menthol and nicotine decreased baseline firing frequency, and following acetylcholine application, neuronal firing frequency increased to levels greater than that found with chronic nicotine treatment alone. Thus, the authors hypothesized that differential nAChR expression may underlie the documented alterations in cellular activity. Indeed, receptor upregulation paralleled the differences found in neuronal excitability. Specifically, while chronic nicotine increased $\alpha 4$ - and $\alpha 6$-containing nAChRs in both the ventral tegmental area and substantia nigra, menthol and nicotine together induced greater upregulation of $\alpha 4$-containing $\mathrm{nAChRs}$ in these regions. Pixel-based FRET further confirmed these findings to support the conclusion that menthol and nicotine cotreatment results in greater expression of $\alpha 4 \alpha 6$-containing nAChRs in the ventral tegmental area.

In sum, these findings emphasize the importance of determining the impact of flavorants and other constituents in tobacco/nicotine products on addiction-related processes. Additional research will be necessary to delineate the interactive effects of nicotine and menthol on the balance of dopaminergic and GABAergic circuit function in vivo, as well as their effects on the neurobiological mechanisms mediating various aspects of dependence, such as reinforcement, withdrawal, and relapse. As the current study provides direct evidence that nicotine and menthol interact on a cellular level to modulate cholinergic signaling mechanisms (Henderson et al, 2017), this interaction may contribute to the disproportionate use and dependence found in certain populations. As such, future policy approaches should take into account the effects of product constituents in the regulation of product availability and marketing to vulnerable populations.

\section{FUNDING AND DISCLOSURE}

CDF is supported by NIH/NIDA grants DA032543 and DA039658. The authors declare no conflict of interest.

\section{REFERENCES}

Fan L, Balakrishna S, Jabba SV, Bonner PE, Taylor SR, Picciotto MR et al (2016). Menthol decreases oral nicotine aversion in C57BL/6 mice through a TRPM8-dependent mechanism. Tob Control 25 (Suppl 2): ii50-ii54.

FDA, 2013. Preliminary scientific evaluation of the possible public health effects of menthol versus nonmenthol cigarettes. Available at: http://www.fda.gov/downloads/UCM361598.pdf. Accessed on April 2017.

Gullota F, Hayes CS, Martin BR, 1989. PREP study on menthol nicotine interactions. Philip Morris. Available at: http://legacy. library.ucsf.edu/tid/bzk48e00.

Henderson BJ, Wall TR, Henley BM, Kim CH, McKinney S, Lester HA (2017). Menthol enhances nicotine reward-related behavior by potentiating nicotine-induced changes in nAChR function, nAChR upregulation, and DA neuron excitability. Neuropsychopharmacology; epub ahead of print 3 May 2017; doi: 10.1038/npp.2017.72.

Henderson BJ, Wall TR, Henley BM, Kim CH, Nichols WA, Moaddel $\mathrm{R}$ et al (2016). Menthol alone upregulates midbrain nAChRs, alters nAChR subtype stoichiometry, alters dopamine neuron firing frequency, and prevents nicotine reward. J Neurosci 36: $2957-2974$.

Villanti AC, Johnson AL, Ambrose BK, Cummings KM, Stanton CA, Rose SW et al (2017). Flavored tobacco product use in youth and adults: findings from the first wave of the PATH study (2013-2014). Am J Prev Med; epub ahead of print 13 March 2017; doi: 10.1016/j.amepre.2017.01.026. 